\title{
Hausse des taux de syphilis au Canada, 2011 à 2020
}

\author{
Josephine Aho ${ }^{1 *}$, Cassandra Lybeck ${ }^{1}$, Ashorkor Tetteh ${ }^{1}$, Carmen Issa ${ }^{1}$, Fiona Kouyoumdjian², \\ Jason Wong ${ }^{3,4}$, Alexandrea Anderson ${ }^{1}$, Nashira Popovic ${ }^{1}$
}

\section{Résumé}

Contexte : Les taux de syphilis constituent un enjeu préoccupant de santé publique au Canada, de nombreuses administrations ayant signalé des éclosions au cours des cinq dernières années. Le présent article vise à décrire les tendances de la syphilis infectieuse et congénitale au Canada de 2011 à 2020.

Méthodes : La surveillance de routine de la syphilis est effectuée par l'entremise du Système canadien de surveillance des maladies à déclaration obligatoire (SCSMDO). En réponse à la hausse des taux de syphilis, toutes les provinces et tous les territoires ont également déclaré à l'Agence de la santé publique du Canada, par l'entremise du Comité de coordination des investigations sur les éclosions de syphilis (CCIES), des données rehaussées sur la surveillance de la syphilis à compter de 2018. Des analyses descriptives des données de surveillance du SCSMDO et du CCIES de 2011 à 2020 selon l'âge, le sexe, l'état de grossesse, l'orientation sexuelle de l'homme et les administrations ont été effectuées.

Résultats : Le taux national de syphilis infectieuse est passé de 5,1 pour 100000 personnes en 2011 à 24,7 pour 100000 personnes en 2020. Les taux ont augmenté dans presque toutes les provinces et territoires $(\mathrm{P} / \mathrm{T})$, les provinces des Prairies ayant déclaré les plus fortes augmentations relatives entre 2016 et 2020 (plus de $400 \%$ ). Les taux chez les hommes étaient constamment plus élevés que ceux rapportés chez les femmes au cours des 10 dernières années; toutefois, de 2016 à 2020, les taux chez les femmes ont augmenté de $773 \%$, comparativement à $73 \%$ chez les hommes. Bien que la proportion de cas qui s'identifient comme gais, bisexuels et autres hommes ayant des rapports sexuels avec des hommes ait diminué de 54 \% à 38 \% entre 2018 et 2020, ils représentent toujours une forte proportion des cas (selon les données de huit P/T). De 2016 à 2020, les taux de syphilis infectieuse ont augmenté dans tous les groupes d'âge, en particulier chez les femmes âgées de 15 à 39 ans. Les cas confirmés de syphilis congénitale précoce pour 2020 ont augmenté considérablement par rapport aux années précédentes, 50 cas ayant été signalés en 2020, comparativement à 4 cas en 2016.

Conclusion : Les taux de syphilis infectieuse et congénitale sont une préoccupation croissante au Canada, et la nature des épidémies de syphilis semble y évoluer, comme le montrent les tendances récentes. Davantage de données et de recherche sont nécessaires pour mieux comprendre les facteurs associés aux changements récents dans l'épidémiologie de la syphilis au Canada.

Citation proposée : Aho J, Lybeck C, Tetteh A, Issa C, Kouyoumdjian F, Wong J, Anderson A, Popovic N. Hausse des taux de syphilis au Canada, 2011 à 2020. Relevé des maladies transmissibles au Canada 2022;48(2/3):58-67. https://doi.org/10.14745/ccdr.v48i23a01f

Mots-clés : syphilis infectieuse, syphilis congénitale, surveillance, gbHARSAH, femmes hétérosexuelles, personnes enceintes, Canada
Cette oeuvre est mise à la disposition selon les termes de la licence internationale Creative Commons Attribution 4.0

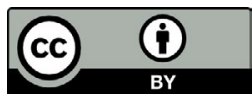

\author{
Affiliations \\ ${ }^{1}$ Centre de la lutte contre les \\ maladies transmissibles et les \\ infections, Agence de la santé \\ publique du Canada, Ottawa, ON \\ 2 Ministère de la Santé de \\ I'Ontario, Toronto, ON \\ ${ }^{3}$ Centre de contrôle des maladies \\ de la Colombie-Britannique, \\ Vancouver, BC \\ ${ }^{4}$ École de santé publique et \\ des populations, Faculté de \\ médecine, Université de la \\ Colombie-Britannique, \\ Vancouver, BC
}

*Correspondance :

josephine.aho@phac-aspc.gc.ca 


\section{Introduction}

La syphilis est la troisième infection sexuellement transmissible à déclaration obligatoire la plus signalée au Canada, après la chlamydia et la gonorrhée. Infection bactérienne causée par la sous-espèce Treponema pallidum, la syphilis se transmet principalement par contact sexuel génital, anal ou oral et peut également se transmettre verticalement au fœtus pendant la grossesse ou au nouveau-né à l'accouchement. La syphilis peut être traitée facilement et efficacement avec de la pénicilline; si elle n'est pas traitée, elle peut évoluer en différents stades, soit primaire, secondaire, de latence précoce, de latence tardive et tertiaire. La syphilis n'est infectieuse que pendant les trois premiers stades de l'infection (primaire, secondaire ou de latence précoce), qui se produisent au cours de la première année. La neurosyphilis aiguë peut également se développer pendant cette période. À des stades plus avancés de la maladie, la syphilis peut entraîner des conséquences graves sur la santé, comme des complications neurologiques, cardiovasculaires ou musculosquelettiques. La syphilis congénitale, c'est-à-dire la syphilis transmise in utero, peut avoir des effets débilitants graves et peut causer une mortinaissance ou un décès néonatal (1).

Après une longue période de diminution des taux de syphilis depuis les années 1940, le Canada a annoncé en 1998 un objectif national visant à maintenir les taux de syphilis en deçà de 0,5 pour 100000 personnes (2). En 2016, I'Organisation mondiale de la Santé a publié des cibles mondiales pour réduire les répercussions des infections sexuellement transmissibles sur la santé d'ici 2030, notamment par une réduction de $90 \%$ des taux de syphilis dans le monde et un seuil de 50 cas ou moins de syphilis congénitale pour 100000 naissances vivantes dans $80 \%$ des pays (3). Le gouvernement du Canada a endossé ces cibles mondiales (4).

Au Canada, les taux de syphilis infectieuse ont commencé à augmenter de façon constante au début des années 2000, avec des augmentations importantes après 2017. Les cas de syphilis congénitale ont également augmenté au cours de la même période. Dix administrations canadiennes ont déclaré des éclosions à l'échelle provinciale, territoriale ou régionale au cours des dernières années (1). En juillet 2019, en réponse à la hausse des taux de syphilis dans l'ensemble des administrations au Canada, I'Agence de la santé publique du Canada (I'Agence), en collaboration avec l'ensemble des provinces et des territoires $(\mathrm{P} / \mathrm{T})$, a mis sur pied un Comité de coordination fédéral, provincial et territorial d'investigation sur les éclosions de syphilis (CCIES). L'objectif du CCIES est d'échanger de I'information sur l'épidémiologie de la syphilis, sur les pratiques exemplaires et sur les défis liés aux réponses à l'augmentation des taux. Toutes les $\mathrm{P} / \mathrm{T}$ partagent des données de surveillance rehaussée sur la syphilis infectieuse confirmée et le nombre de cas confirmés de syphilis congénitale précoce directement à l'Agence dans le cadre des activités du CCIES.
Dans cet article, les tendances récentes des taux de syphilis infectieuse et congénitale au Canada sont décrites à l'aide des données de surveillance de 2011 à 2020.

\section{Méthodes}

\section{Source de données}

Depuis 1924, les autorités sanitaires provinciales et territoriales ont fournis régulièrement à l'Agence des données sur les cas de syphilis confirmés en laboratoire par l'entremise du Système canadien de surveillance des maladies à déclaration obligatoire (SCSMDO) (5). Bien que tous les stades de la syphilis soient à déclaration obligatoire, seuls les cas infectieux confirmés et les cas congénitaux précoces confirmés ont été inclus dans notre analyse. Les définitions nationales des cas de tous les stades de la syphilis ainsi que de la syphilis congénitale se trouvent en ligne (6).

Les variables relatives aux cas soumises au SCSMDO comprenaient le sexe, l'âge au moment du diagnostic, I'année du diagnostic et la P/T du diagnostic, tandis que les variables soumises au CCIES comprenaient également l'orientation sexuelle pour les hommes et l'état de grossesse. Toutes les P/T ont soumis des données sur le sexe, l'âge et I'année du diagnostic au SCSMDO et au CCIES. Les données de Terre-Neuve-et-Labrador ont été stratifiées selon l'âge et le sexe, mais pas selon l'âge et le sexe simultanément. Pour les années 2018 à 2020, la Colombie-Britannique, l'Alberta, la Saskatchewan, l'Ontario, le Nouveau-Brunswick, la Nouvelle-Écosse, le Yukon et les Territoires du Nord-Ouest ont systématiquement rapporté des données sur l'orientation sexuelle des hommes et l'état de grossesse au CCIES. Ces huit provinces et territoires représentaient $72 \%$ de la population canadienne. Les cas confirmés de syphilis congénitale précoce (moins de deux ans après la naissance) ne comprenaient pas les mortinaissances.

Les données des années précédant la collecte des données du CCIES (2011 à 2017) ont été extraites du SCSMDO, tandis que les données de 2018 à 2020 ont été recueillies au moyen du CCIES. Douze provinces et territoires ont soumis des données pour l'année civile complète de 2020, et une province (Terre-Neuve-et-Labrador) a fourni des chiffres partiels pour 2020; par conséquent, les chiffres annuels pour 2020 pour cette province ont été extrapolés.

\section{Analyse des données}

Le nombre de cas, les proportions et les taux sont présentés dans l'ensemble et selon le sexe, l'orientation sexuelle de I'homme, le groupe d'âge et la P/T. Le nombre de cas de personnes enceintes est également inclus. Pour ce qui est de la déclaration des données par les $\mathrm{P} / \mathrm{T}$, la proportion de données manquantes pour le sexe et l'âge ne dépassait pas $1 \%$, tandis qu'elle variait de $0 \%$ à $18 \%$ pour les données sur 
I'orientation sexuelle des hommes et l'état de grossesse. Les taux de syphilis infectieuse confirmée et de syphilis congénitale précoce confirmée ont été calculés à l'aide des nombres de cas déclarés comme numérateurs et des estimations de la population et des naissances vivantes de Statistique Canada, mises à jour pour la dernière fois en juillet 2020, comme dénominateurs. Les taux déclarés ont été calculés pour 100000 personnes et comprenaient les taux annuels globaux ainsi que les taux par sexe, groupe d'âge et $\mathrm{P} / \mathrm{T}$. Des analyses descriptives ont été effectuées à l'aide de la version R 4.0.2 (7-9) et de Microsoft Excel. Les tendances observées basées sur de petits nombres doivent être interprétées avec prudence, car les taux calculés à partir de petits nombres sont plus susceptibles de fluctuer au fil du temps.

\section{Résultats}

Depuis 2011, il y a eu une augmentation constante du nombre de cas confirmés de syphilis infectieuse signalés, à part une légère baisse en 2020 (figure 1). Les augmentations les plus élevées ont été observées en 2018 et en 2019 (augmentations annuelles de $50 \%$ et de $45 \%$, respectivement), ce qui correspond au nombre de cas et aux taux les plus élevés enregistrés au cours des 10 dernières années. On estime qu'il y a eu 9382 cas en 2020, ce qui correspond à un taux de 24,7 pour 100000 personnes (figure 1). Cela reflète une augmentation de $385 \%$ des taux par rapport au taux de 5,1 pour 100000 personnes en 2011.

Figure 1 : Nombre total de cas et taux de syphilis infectieuse selon le sexe au Canada par année, 2011 à 2020

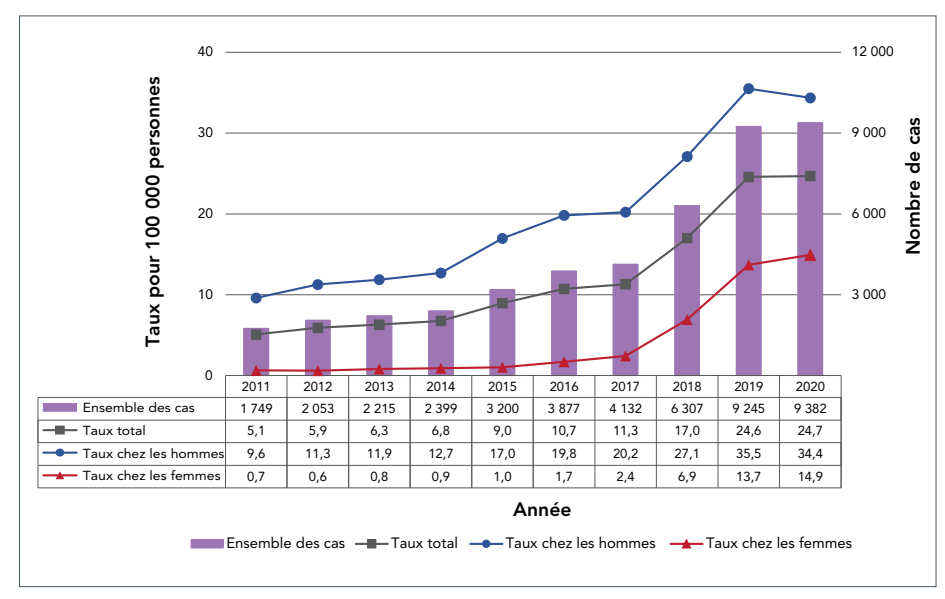

\section{Les taux de syphilis infectieuse ont augmenté chez les hommes et les femmes, mais plus rapidement chez les femmes}

Au cours de la dernière décennie, le taux annuel de syphilis infectieuse a toujours été plus élevé chez les hommes que chez les femmes (figure 1). Les taux de syphilis infectieuse ont augmenté tant chez les hommes que chez les femmes de 2011 à 2020; cependant, l'ampleur de la hausse a été plus prononcée chez les femmes, surtout au cours des cinq dernières années. De 2016 à 2020, les taux ont augmenté de $73 \%$ chez les hommes, mais de 773 \% chez les femmes. Néanmoins, en 2020, le taux chez les hommes est demeuré plus élevé que chez les femmes, à 34,4 pour 100000 personnes comparativement à 14,9 pour 100000 personnes.

La proportion de tous les cas de syphilis infectieuse signalés chez les femmes a également augmenté considérablement. La proportion nationale de cas de syphilis infectieuse chez les femmes était de $6 \%$ en 2011 et est demeurée inférieure à 10 \% jusqu'en 2017. En 2019 et en 2020, la proportion de tous les cas de syphilis infectieuse signalés chez les femmes était de $28 \%$ et de $30 \%$ respectivement. En 2020, les P/T affichant les proportions les plus élevées de cas de femmes étaient la Saskatchewan (51 \%), le Manitoba (49\%) et l'Alberta (43\%). Bien que le nombre de cas soit faible (50 cas ou moins), le Nunavut a également déclaré une forte proportion de cas de sexe féminin (60\%). La Colombie-Britannique, I'Ontario et le Québec ont déclaré les plus faibles proportions de cas de femmes en 2020 (inférieur ou égal à $15 \%$ ).

\section{La proportion de cas de syphilis infectieuse demeure la plus élevée chez les gais, les bisexuels et les autres hommes qui ont des rapports sexuels avec des hommes, mais elle diminue}

Les huit $\mathrm{P} / \mathrm{T}$ qui ont déclaré l'orientation sexuelle des hommes représentaient $71 \%$ de tous les cas d'hommes signalés au pays en 2020. Selon les données de 2020 fournies par ces provinces et territoires, $38 \%$ de tous les cas de syphilis infectieuse ont été signalés chez des hommes gais, bisexuels et autres hommes ayant des rapports sexuels avec des hommes (gbHARSAH), et $25 \%$ chez des hommes hétérosexuels. À titre de comparaison, en 2018 , les huit mêmes $\mathrm{P} / \mathrm{T}$ ont déclaré une proportion de $54 \%$ des cas s'identifiant comme des hommes gbHARSAH et de $24 \%$ d'hommes hétérosexuels.

\section{Les taux de syphilis infectieuse augmentent plus rapidement chez les jeunes adultes}

Dans l'ensemble, les taux de syphilis infectieuse ont augmenté entre 2011 et 2019 dans presque tous les groupes d'âge, mais sont demeurés relativement inchangés de 2019 à 2020 (figure 2). Le taux le plus élevé en 2020 a été observé chez les 25 à 29 ans (69,8 pour 100000 personnes).

Pour toutes les années d'intérêt (2011 à 2020), les taux chez les hommes étaient toujours les plus élevés chez les 25 à 29 ans (85,5 pour 100000 personnes en 2020), suivis de près par les 30 à 39 ans (82,3 pour 100000 en 2020) (figure 2A). 
Figure 2 : Taux de syphilis infectieuse chez les hommes et les femmes pour 100000 personnes par groupe d'âge au Canada, 2011 à 2020 a,b

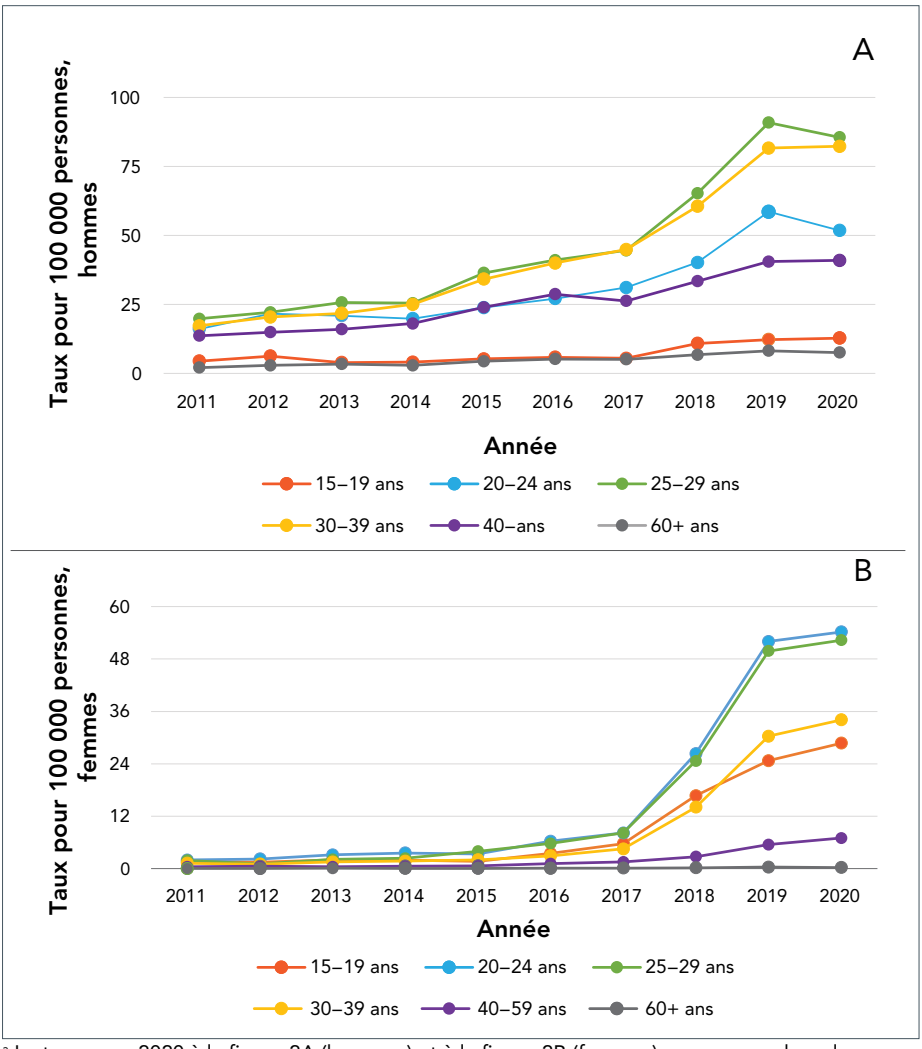

Le taux pour 2020 à la figure 2A (hommes) et à la figure 2B (femmes) ne comprend pas les données pour Terre-Neuve-et-Labrador, car la province n'a pas fourni de données stratifiées par sexe ET par âge pour cette année

Veuillez noter que les échelles des figures $2 \mathrm{~A}$ et $2 \mathrm{~B}$ diffèren

Les taux de syphilis infectieuse chez les femmes étaient les plus élevés dans les groupes plus jeunes comparativement à leurs homologues masculins (figure 2B). Jusqu'en 2017, on a observé une augmentation constante des taux de syphilis infectieuse chez les femmes, principalement chez les 15 à 39 ans. En 2018, les taux chez les femmes de cette tranche d'âge ont triplé (en hausse de plus de $200 \%$ par rapport à l'année précédente). En 2020, les taux les plus élevés de syphilis infectieuse chez les femmes ont été observés chez les personnes âgées de 20 à 24 ans (54,2 pour 100000 personnes). Bien que les taux ne soient que légèrement supérieurs à ceux observés chez les hommes du même groupe d'âge (51,8 pour 100000 personnes), c'est la première fois au cours de la période d'intérêt (2011 à 2020) que les taux chez les femmes de ce groupe d'âge dépassent ceux des hommes. Le deuxième taux le plus élevé de syphilis infectieuse chez les femmes a été signalé chez les femmes de 25 à 29 ans (52,3 pour 100000 personnes).

La proportion de tous les cas de syphilis infectieuse signalés chez les femmes âgées de 15 à 39 ans a également augmenté rapidement. En 2011, 5,2 \% de tous les cas ont été signalés chez des femmes âgées de 15 à 39 ans, ce qui a quintuplé pour atteindre environ le quart (26\%) des cas en 2020. De 2016 à 2020 (figure 2B), une augmentation de $858 \%$ des taux de syphilis infectieuse a été observée chez les femmes âgées de 15 à 39 ans. En 2020, les femmes de 15 à 39 ans représentaient $87 \%$ de tous les cas chez les femmes.

\section{Les taux de syphilis infectieuse augmentent dans presque toutes les provinces et tous les territoires}

En 2011, neuf P/T ont déclaré des cas de syphilis infectieuse, avec des taux variant de 1,0 pour 100000 personnes à Terre-Neuve-et-Labrador à 7,8 pour 100000 personnes au Québec. Depuis, les taux ont augmenté de façon constante dans la plupart des $\mathrm{P} / \mathrm{T}$, et d'importantes augmentations ont été observées en 2017 et en 2018. De 2016 à 2020, les provinces des Prairies ont déclaré les plus fortes augmentations relatives (plus de $400 \%$ ). Dans la plupart des P/T, les taux les plus élevés de syphilis infectieuse signalés au cours des 10 dernières années ont été enregistrés en 2019. Les $\mathrm{P} / \mathrm{T}$ affichant les taux les plus élevés de syphilis infectieuse en 2019 étaient le Nunavut (266,7 pour 100000 personnes), le Manitoba (136,7 pour 100000 personnes), les Territoires du Nord-Ouest (97,7 pour 100000 personnes), l'Alberta (51,9 pour 100000 personnes) et la Saskatchewan (33,7 pour 100000 personnes). Les taux ont diminué de 2019 à 2020 au Manitoba, dans les Territoires du Nord-Ouest et au Nunavut, mais ont augmenté en Alberta et en Saskatchewan (figure 3).

Figure 3 : Taux de cas déclarés de syphilis infectieuse au Canada, par province et territoire, 2020

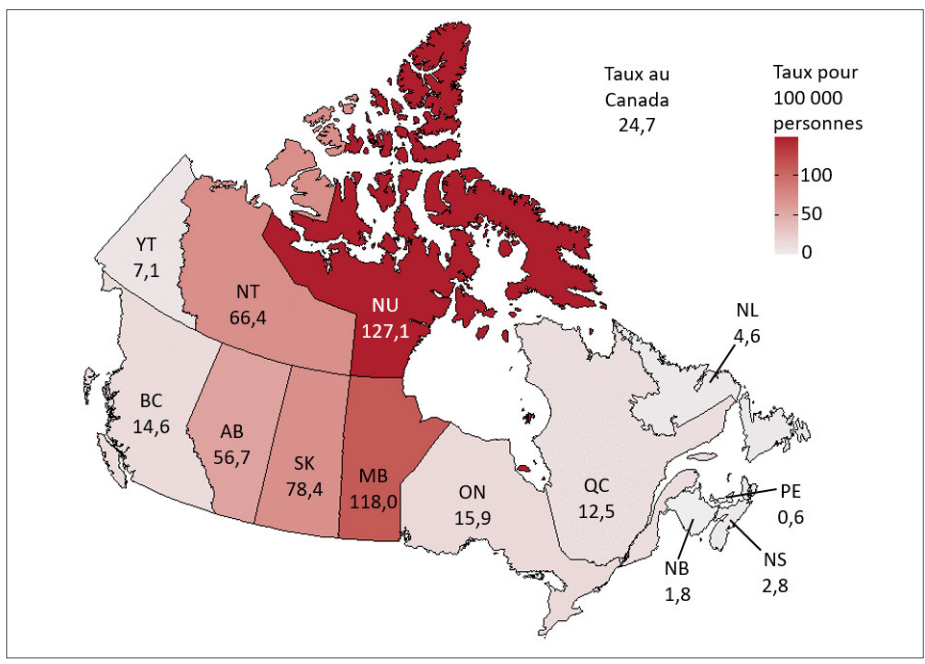

Abréviations : $A B$, Alberta; $B C$, Colombie-Britannique; $P E$, Île-du-Prince-Édouard; MB, Manitoba NB, Nouveau-Brunswick; NS, Nouvelle-Écosse; NU, Nunavut; ON, Ontario; QC, Québec; SK, Saskatchewan; NL, Terre-Neuve-et-Labrador; NT, Territoires du Nord-Ouest; YT, Yukon

\section{Les cas précoces de syphilis congénitale sont également en hausse}

Le nombre déclaré de cas confirmés de syphilis congénitale précoce a augmenté au cours des 10 dernières années au Canada (figure 4). De 2011 à 2017, 10 cas ou moins de syphilis congénitale précoce confirmée ont été observés par année. 
Les cas déclarés de syphilis congénitale précoce confirmée ont plus que doublé entre 2017 et 2018, passant de 7 cas en 2017 à 17 cas en 2018. En 2019 et en 2020, le nombre total de cas a augmenté à 53 et 50 , respectivement, ce qui correspond à un taux de 14,2 pour 100000 naissances vivantes en 2019 et de 13,4 pour 100000 naissances vivantes en 2020 .

Figure $4:$ Nombre et taux de cas confirmés de syphilis congénitale précoce déclarés pour 100000 naissances vivantes au Canada, 2011 à 2020

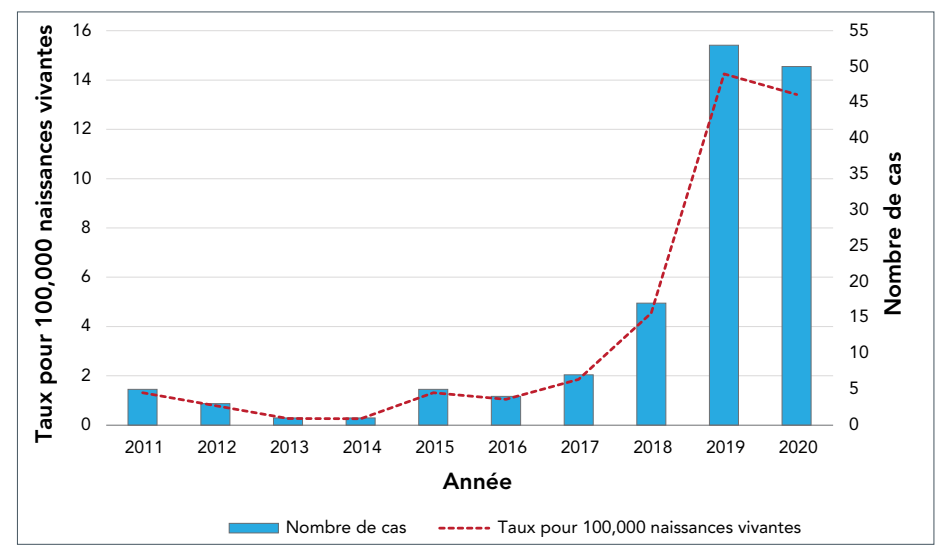

En 2019, I'Alberta et le Manitoba ont déclaré $79 \%$ de tous les cas confirmés de syphilis congénitale précoce au Canada, tandis que les autres $\mathrm{P} / \mathrm{T}$ ont déclaré trois cas ou moins de syphilis congénitale. En 2020, la majorité ( $86 \%$ ) des cas confirmés de syphilis congénitale précoce signalés ont été observés en Alberta, au Manitoba et en Saskatchewan. Ces P/T affichaient également les taux les plus élevés de syphilis infectieuse chez les femmes de 15 à 39 ans.

Parmi les huit $\mathrm{P} / \mathrm{T}$ qui ont soumis des données sur l'état de la grossesse, 230 cas de syphilis infectieuse ont été signalés chez des personnes enceintes en 2020. Les mêmes $\mathrm{P} / \mathrm{T}$ ont signalé 71 cas de syphilis infectieuse chez les personnes enceintes en 2018 et 133 cas en 2019.

\section{Discussion}

Les taux de syphilis infectieuse au Canada ont augmenté considérablement au cours de la dernière décennie, surtout depuis 2017. À l'instar des États-Unis, du Royaume-Uni et de l'Australie, la majorité des cas (selon des données partielles) au Canada se sont produits chez les hommes, en particulier chez les gbHARSAH (10-12). Cependant, bien que les taux au Canada soient demeurés plus élevés chez les hommes que chez les femmes, les taux de syphilis infectieuse chez les femmes ont augmenté rapidement au cours des dernières années. Des tendances semblables ont également été observées dans d'autres pays. Les États-Unis, le Royaume-Uni et l'Australie ont tous signalé des taux croissants de syphilis infectieuse, les taux ayant augmenté de plus de $175 \%$ entre 2015 et 2019 , et les taux chez les femmes ayant augmenté plus rapidement que chez les hommes (10-12).

Les raisons qui expliquent les taux élevés de syphilis chez les hommes, et en particulier chez les hommes de la communauté gbHARSAH, et les augmentations plus récentes chez les populations hétérosexuelles demeurent nébuleuses, car les données sur les comportements sexuels et sur les populations clés sont incomplètes dans le cadre de la surveillance nationale. Les déterminants sociaux et structurels de la santé et les inégalités en santé jouent sans aucun doute un rôle crucial dans l'apparition inéquitable de la syphilis dans différentes populations $(1,13)$. Ces déterminants comprennent l'instabilité du logement, les expériences de violence, le manque d'accès à des soins culturellement adaptés et les expériences de stigmatisation, de discrimination et de racisme, en particulier au sein du système de santé $(1,14-19)$.

Les données disponibles au Canada et aux États-Unis suggèrent que la consommation de substances pourrait contribuer à expliquer les tendances récentes en matière de taux de syphilis. La consommation de substances, y compris les opioïdes et la méthamphétamine en cristaux (" crystal meth »), est devenue plus fréquente ces dernières années (20-23). Bien que la prévalence de la consommation de méthamphétamine en cristaux soit faible dans l'ensemble au Canada (moins de $1 \%$ de la population générale et 11 fois moins qu'aux États-Unis), sa disponibilité, sa consommation et les méfaits qui y sont associés ont considérablement augmenté depuis 2013 (20). La consommation de méthamphétamine en cristaux peut influencer la prise de décisions sur les pratiques sexuelles à moindre risque $(24,25)$. Chez la population gbHARSAH, le « chemsex ", soit l'utilisation de substances (comme le crystal meth) pour prolonger et intensifier les expériences sexuelles, a été décrit comme une tendance majeure $(24,26,27)$. Il a été démontré que l'utilisation de drogues par injection et la consommation problématique d'alcool chez la population gbHARSAH sont des comportements qui augmentent le risque de co-infection par la syphilis et le virus de l'immunodéficience humaine (28-30). La consommation de méthamphétamine en cristaux a également été un comportement signalé lors d'une éclosion concernant des personnes hétérosexuelles à Winnipeg en 2017-2018 et a été identifiée comme un facteur en 2019 pour l'éclosion de syphilis en Saskatchewan (16,31). Des données récentes des États-Unis montrent également des associations entre la consommation de méthamphétamine en cristaux, la consommation d'opioïdes et des éclosions de syphilis dans les populations hétérosexuelles (32-34).

Au cours des 10 dernières années, les femmes âgées de 15 à 39 ans représentaient en moyenne $96 \%$ des mères de naissances vivantes au Canada (35). De 2016 à 2020, une augmentation 
de près de 10 fois les cas de syphilis infectieuse (858 \%) a été signalée chez les femmes âgées de 15 à 39 ans, et ce groupe représente $87 \%$ de tous les cas de syphilis chez les femmes en 2020. L'augmentation des cas de syphilis infectieuse chez les femmes de ce groupe d'âge a entraîné une augmentation subséquente des cas de syphilis congénitale au Canada. Un nombre record de cas confirmés de syphilis congénitale précoce ont été signalés en 2019 et en 2020 (au moins 50 cas chaque année, comparativement à 10 cas ou moins avant 2017). II s'agit du nombre le plus élevé de cas confirmés de syphilis congénitale précoce signalés depuis que la syphilis congénitale est devenue une maladie à déclaration obligatoire en 1993. Les États-Unis ont également connu une situation semblable : 1870 cas de syphilis congénitale ont été déclarés en 2019 , soit une augmentation de 291 \% par rapport à 2015 (10). Les femmes sont confrontées à des défis uniques en matière de diagnostic précoce en raison de différences anatomiques ou biologiques susceptibles de retarder l'identification des lésions primaires (1). Ce facteur peut contribuer à retarder les tests et l'accès aux soins précoces. II convient de noter que certaines administrations ont modifié leurs lignes directrices de pratique clinique sur le dépistage prénatal au cours de la dernière décennie afin d'augmenter la fréquence des tests de dépistage de la syphilis au cours d'une grossesse (36-42). Cette modification des lignes directrices peut avoir entraîné un dépistage plus uniforme et plus fréquent de la syphilis - et une plus grande détection des cas - chez les femmes enceintes et en âge de procréer en général, comparativement aux tests pour les hommes. Par ailleurs, des études menées aux États-Unis ont indiqué que les facteurs potentiels associés à l'augmentation des taux de syphilis pendant la grossesse et de syphilis congénitale comprenaient le manque d'accès aux soins prénataux et l'absence d'occasions de traitement rapide et efficace pour les femmes enceintes $(43,44)$.

Divers obstacles structurels, notamment le faible revenu, I'insécurité du logement et la résidence en milieu rural ou éloigné, ont été associés aux cas d'infection par la syphilis, particulièrement pendant la grossesse $(1,36,45-47)$. De plus, la discrimination systémique, le racisme et la stigmatisation dans les milieux de soins de santé en raison de la consommation de substances peuvent contribuer à la méfiance à l'égard des systèmes de soins de santé et constituer des obstacles aux soins pour les personnes ayant la syphilis $(1,13,43,48,49)$. Au moins une province a signalé de manière anecdotique des diagnostics plus fréquents de stades latents, ce qui indique que des diagnostics ont été omis à des stades plus précoces de I'infection, peut-être en raison d'obstacles structurels dans les soins. Les tests de dépistage rapide qui peuvent être effectués à l'endroit où des soins sont donnés aux patients, comme les tests au point de soin, peuvent jouer un rôle important pour le contrôle de la syphilis dans les populations éloignées ou marginalisées $(50,51)$. Toutefois, aucun test de dépistage de la syphilis au point de soins n'a été autorisé pour utilisation au Canada $(1,52)$.
Les taux de syphilis infectieuse signalés dans le présent article peuvent sous-estimer les taux réels, car les personnes vivant avec l'infection n'ont pas nécessairement accès aux soins de santé ou aux services de dépistage des infections transmissibles sexuellement et par le sang (ITSS). Cette sous-estimation pourrait notamment s'être produite pendant la pandémie de la maladie à coronavirus de 2019 (COVID-19) en raison des mesures de santé publique visant à contrôler la pandémie et limitant l'accès aux services liés aux ITSS. Comme la pandémie a été déclarée en mars 2020, les diminutions observées en 2020 pourraient ne pas refléter l'incidence réelle. Un sondage en ligne mené par l'Agence en 2021 a montré que la pandémie de COVID-19 a eu une incidence sur la détection des ITSS et les interventions subséquentes (53). L'enquête a révélé que la pandémie a entraîné une diminution de la demande et de la capacité à offrir des services de prévention, de dépistage et de traitement des ITSS, mais une augmentation de la demande de services de réduction des méfaits et de traitement des dépendances (53). L'incidence de la pandémie sur la surveillance des ITSS a également été signalée dans d'autres pays, ce qui souligne la diminution du dépistage et des tests et les ressources limitées, entraînant ainsi un sous-diagnostic et une sous-déclaration (54-56).

Compte tenu de la perturbation des services et des rapports sur l'augmentation de la consommation de substances, l'incidence de la pandémie sur l'incidence réelle de la syphilis reste à déterminer. Cela pourrait se refléter dans les données de surveillance nationale de 2021 et de 2022, selon l'évolution de la pandémie de COVID-19 et son incidence sur le dépistage des ITSS $(53,57)$. Il est important de noter que le système national de surveillance rehaussé comporte des limites, comme des données incomplètes sur des variables incluant l'orientation sexuelle des hommes et la consommation de substances (cette dernière variable $n^{\prime}$ est pas présentée dans cet article en raison des niveaux élevés de données manquantes) qui ne sont pas recueillies de façon systématique et uniforme dans l'ensemble des provinces et territoires. De plus, la collecte de données et la recherche sur d'autres déterminants sociaux de la santé, notamment les données sur l'ethnicité et sur l'indigénéité, seraient essentielles pour mieux comprendre les épidémies, bien que la collecte de données sur l'identité ethnoculturelle demeure controversée. Néanmoins, il y a un consensus croissant, surtout à la lumière des leçons tirées de la pandémie de COVID-19, selon lequel les données désagrégées sont importantes pour mieux décrire les épidémies et cibler adéquatement les interventions (58-60).

\section{Conclusion}

Cet article brosse un portrait national de l'épidémie de syphilis au Canada au cours des 10 dernières années et traite des facteurs potentiels associés aux augmentations récentes considérables des taux de syphilis. L'incidence de la syphilis a grimpé en flèche récemment, et la nature des épidémies au Canada semble varier et évoluer, avec un effet persistant sur la 
population gbHARSAH et un nombre croissant d'éclosions chez les hommes et les femmes hétérosexuels. Le nombre de cas de syphilis congénitale a également augmenté considérablement. Une meilleure compréhension des facteurs sociaux, structurels et comportementaux qui influent sur la syphilis pourrait aider à déterminer les possibilités d'interventions à l'aide d'une approche syndémique. II faut poursuivre la recherche pour comprendre l'étendue des éclosions de syphilis et des déterminants qui y sont associés au Canada. De telles données pourraient éclairer les efforts visant à réduire l'impact des ITSS sur la santé et à atteindre l'objectif mondial de l'Organisation mondiale de la Santé visant à réduire l'incidence de la syphilis d'ici 2030.

\section{Déclaration des auteurs}

J. A. - Conceptualisation, rédaction de la version préliminaire, révision de la rédaction et édition, supervision

C. L. - Conservation, validation, visualisation, analyse des données, analyse documentaire, rédaction de la version préliminaire, révision de la rédaction et édition

A. T. - Rédaction de la version préliminaire, analyse documentaire, révision de la rédaction et édition, validation C. I. - Collecte et conservation des données, validation, analyse des données, analyse documentaire, rédaction de la version préliminaire, révision de la rédaction et édition

F. K. - Révision de la rédaction et édition

J. W. - Révision de la rédaction et édition

A. A. - Collecte des données, révision de la rédaction et édition N. P. - Conceptualisation, révision de la rédaction et édition

\section{Intérêts concurrents}

Aucun.

\section{Remerciements}

Les auteurs remercient B. Ephrem, G. Gravel, M.-A. Leblanc, R. Naraine, M. Sabourin, S. Sabourin, R. Tsang et J. Wu pour leurs points de vue, leurs conseils et leurs révisions pendant I'examen de cet article. Les auteurs remercient également S. Thomas d'avoir contribué au résumé initial, J. Sandhu d'avoir aidé à la gestion des références et l'équipe de la bibliothèque de Santé Canada et de l'Agence de la santé publique du Canada d'avoir aidé à la recherche documentaire de l'article. Enfin, les auteurs sont reconnaissants envers toutes les provinces et tous les territoires pour leur contribution à l'établissement du portrait national des données de surveillance améliorées sur la syphilis au Canada de 2011 à 2020 et pour leur révision de l'article.

\section{Financement}

Ce travail a été appuyé par l'Agence de la santé publique du Canada.

\section{Références}

1. Agence de la santé publique du Canada. La syphilis au Canada: rapport technique sur les tendances épidémiologiques, les déterminants et interventions. Ottawa (ON) : ASPC; 2020. https://www.canada.ca/fr/services/ sante/publications/maladies-et-affections/syphilis-rapportepidemiologiques.html

2. Santé Canada. Compte rendu de la Réunion nationale et concertation sur les MTS et Objectifs nationaux en matière de prévention et de contrôle des maladies transmises sexuellement au Canada. Relevé des maladies transmissibles au Canada 1997;23 S6:1-27. https://publications.gc.ca/ collections/collection_2016/aspc-phac/HP3-1-23-S6-fra.pdf

3. Organisation mondiale de la Santé. Stratégie mondiale du secteur de la santé contre les infections sexuellement transmissibles 2016-2021. Genève (CH) : OMS; 2016. https://www.who.int/reproductivehealth/publications/rtis/ ghss-stis/fr/

4. Agence de la santé publique du Canada. Réduction des répercussions sur la santé des infections transmissibles sexuellement et par le sang au Canada d'ici 2030 : un cadre d'action pancanadien sur les ITSS. Ottawa (ON) : ASPC; 2018. https://www.canada.ca/fr/sante-publique/ services/maladies-infectieuses/sante-sexuelle-infectionstransmissibles-sexuellement/rapports-publications/ infections-transmissibles-sexuellement-sang-cadre-action. html

5. Agence de la santé publique du Canada. Graphiques de maladies à déclaration obligatoire. Ottawa (ON) : ASPC; 2021. https://maladies.canada.ca/declaration-obligatoire/ liste-graphiques

6. Agence de la santé publique du Canada. Définitions de cas: maladies à déclaration obligatoire à l'échelle nationale. Ottawa (ON) : ASPC; 2008. https://maladies.canada.ca/ declaration-obligatoire/liste-maladies

7. R Core Team. The R Project for Statistical Computing. Vienna, Austria. http://R-project.org/

8. Package Documentation R. mccormackandrew/ mapcan: Tools for Plotting Canadian Choropleth Maps and Choropleth Alternatives. https://rdrr.io/github/ mccormackandrew/mapcan/

9. Wickham $\mathrm{H}$, Averick M, Bryan J, Chang W, D'Agostino McGowan L, François R, Grolemund G, Hayes A, Henry L, Hester J, Kuhn M, Pedersen TL, Miller E, Milton Bache S, Muller K, Ooms J, Robinson D, Seidel DP, Spinu V, Takahashi K, Vaughan D, Wilke C, Woo K, Yutani H. Welcome to the Tidyverse. J Open Source Softw 2019;4(43):1686. DOI 
10. Centers for Disease Control and Prevention. National Overview - Sexually Transmitted Disease Surveillance, 2019. Atlanta (GA): CDC; 2021. https://www.cdc.gov/std/ statistics/2019/overview.htm

11. Public Health England. Tracking the syphilis epidemic in England: 2010 to 2019. London, UK; Public Health England; 2021. https://assets.publishing.service.gov.uk/government/ uploads/system/uploads/attachment_data/file/956716/ Syphilis_Action_Plan_Metrics_2010_to_2019_report.pdf

12. Kirby Institute, University South Wales. Sexually transmissible infections. Sydney, Australia: UNSW; 2020. https://data. kirby.unsw.edu.au/STIs

13. National Academies of Sciences, Engineering, and Medicine. Sexually Transmitted Infections: Adopting a Sexual Health Paradigm (2021). Washington (DC): The National Academies Press, 2021. https://www.nap.edu/read/25955/chapter/1

14. Johnson L. Worst since 1948: Edmonton the epicentre of syphilis outbreak declared in Alberta. Edmonton Journal. July 16, 2019. https://edmontonjournal.com/news/localnews/infectious-syphilis-outbreak-declared-in-alberta

15. Northwestern Health Unit. Syphilis Epidemiologic Summary. July 30, 2019. Ontario: NWHU; 2019. https://www2. nwhu.on.ca/wp-content/uploads/2022/01/Syphilis-EpiSummary-2019-07-30.pdf

16. Shaw S, Lapple A, Reimer J, Ross C, Nowicki D, Elliott L, Plourde P. P768 The evolution of an infectious syphilis epidemic in a Canadian urban setting. Sex Transm Infect 2019;95 Suppl 1:A330. DOI

17. Ferlatte O, Salway T, Samji H, Dove N, Gesink D, Gilbert M, Oliffe JL, Grennan T, Wong J. An Application of Syndemic Theory to Identify Drivers of the Syphilis Epidemic Among Gay, Bisexual, and Other Men Who Have Sex With Men. Sex Transm Dis 2018;45(3):163-8. DOI PubMed

18. Seth P, Raiford JL, Robinson LS, Wingood GM, Diclemente RJ. Intimate partner violence and other partner-related factors: correlates of sexually transmissible infections and risky sexual behaviours among young adult African American women. Sex Health 2010;7(1):25-30. DOI PubMed

19. Andermann A. Les éclosions dans l'optique de la syndémie : de nouvelles notions pour améliorer la santé des Autochtones. Relevé des maladies transmissibles au Canada. 2017;43(6):140-8. DOI

20. Centre canadien sur les dépendances et l'usage de substances. Méthamphétamine (Sommaire canadien sur la drogue). Ottawa (ON): CCDUS; 2020. https://www.ccsa.ca/ $\mathrm{fr} /$ methamphetamine-sommaire-canadien-sur-la-drogue

21. Fischer B, O'Keefe-Markman C, Lee AM, Daldegan-Bueno D. 'Resurgent', 'twin' or 'silent' epidemic? A select data overview and observations on increasing psycho-stimulant use and harms in North America. Subst Abuse Treat Prev Policy 2021;16(1):17. DOI PubMed
22. Tarasuk J, Zhang J, Lemyre A, Cholette F, Bryson M, Paquette $D$. Résultat nationaux de l'enquête Track auprès des utilisateurs de drogues injectables au Canada, phase 4, 2017 à 2019. Relevé des maladies transmissibles au Canada 2020;46(5):155-68. DOI

23. Centre canadien sur les dépendances et l'usage de substances. Bulletin du RCCET : Changements dans I'usage de stimulants et ses méfaits : gros plan sur la méthamphétamine et la cocaïne. Ottawa (ON) : CCDUS; 2019. https://www.ccsa.ca/sites/default/files/2019-05/CCSACCENDU-Stimulant-Use-Related-Harms-Bulletin-2019-fr.pdf

24. Maxwell S, Shahmanesh M, Gafos M. Chemsex behaviours among men who have sex with men: A systematic review of the literature. Int J Drug Policy 2019;63:74-89. DOI PubMed

25. Singh AE. How to address the resurgence of syphilis in Canada. CMAJ 2019;191(50):E1367-8. DOI

26. Flores Anato JL, Panagiotoglou D, Greenwald ZR, Trottier C, Vaziri M, Thomas R, Maheu-Giroux M. Chemsex practices and pre-exposure prophylaxis (PrEP) trajectories among individuals consulting for PrEP at a large sexual health clinic in Montréal, Canada (2013-2020). Drug Alcohol Depend 2021;226:108875. DOI PubMed

27. Guerra FM, Salway TJ, Beckett R, Friedman L, Buchan SA. Review of sexualized drug use associated with sexually transmitted and blood-borne infections in gay, bisexual and other men who have sex with men. Drug Alcohol Depend 2020;216:108237. DOI PubMed

28. Lang R, Read R, Krentz HB, Ramazani S, Peng M, Gratrix J, Gill MJ. Increasing incidence of syphilis among patients engaged in HIV care in Alberta, Canada: a retrospective clinic-based cohort study. BMC Infect Dis 2018;18(1):125. DOl PubMed

29. Burchell AN, Allen VG, Gardner SL, Moravan V, Tan DH, Grewal R, Raboud J, Bayoumi AM, Kaul R, Mazzulli T, McGee F, Rourke SB; OHTN Cohort Study Team. High incidence of diagnosis with syphilis co-infection among men who have sex with men in an HIV cohort in Ontario, Canada. BMC Infect Dis 2015;(15):356. DOI PubMed

30. Remis RS, Liu J, Loutfy MR, Tharao W, Rebbapragada A, Huibner S, Kesler M, Halpenny R, Grennan T, Brunetta J, Smith G, Reko T, Kaul R. Prevalence of Sexually Transmitted Viral and Bacterial Infections in HIV-Positive and HIV-Negative Men Who Have Sex with Men in Toronto. PLoS One 2016;11(7):e0158090. DOI PubMed

31. Vescera Z. Sask. syphilis outbreak continues as doctors struggle to find patients. Saskatoon StarPhoenix December 19, 2019. https://thestarphoenix.com/news/localnews/sask-syphilis-outbreak-continues-as-doctors-struggleto-find-patients

32. Kidd SE, Grey JA, Torrone EA, Weinstock HS. Increased Methamphetamine, Injection Drug, and Heroin Use Among Women and Heterosexual Men with Primary and Secondary Syphilis - United States, 2013-2017. MMWR Morb Mortal Wkly Rep 2019;68(6):144-8. DOI PubMed 
33. Centers for Disease Control and Prevention (CDC). Methamphetamine use and HIV risk behaviors among heterosexual men--preliminary results from five northern California counties, December 2001-November 2003. MMWR Morb Mortal Wkly Rep 2006;55(10):273-7. PubMed

34. Flom PL, Friedman SR, Kottiri BJ, Neaigus A, Curtis R, Des Jarlais DC, Sandoval M, Zenilman JM. Stigmatized drug use, sexual partner concurrency, and other sex risk network and behavior characteristics of 18- to 24-year-old youth in a high-risk neighborhood. Sex Transm Dis 2001;28(10):598-607. DOI PubMed

35. Statistique Canada. Tableau : 13-10-0416-01: Naissances vivantes, selon l'âge de la mère. Ottawa $(\mathrm{ON})$ : StatCan; 2021. https://www150.statcan.gc.ca/t1/tb/1/fr/tv.action?pid $=1310041601 \&$ request_locale $=$ fr

36. Plitt SS, Osman M, Sahni V, Lee BE, Charlton C, Simmonds K. Examination of a prenatal syphilis screening program, Alberta, Canada: 2010-2011. Can J Public Health 2016;107(3):e285-90. DOI PubMed

37. Government of Nunavut. Nunavut Communicable Disease and Surveillance Manual. Iqaluit (NU): Government of Nunavut; 2018. https://www.gov.nu.ca/sites/default/files/nu_ communicable_diseases_manual_-_complete_2018-_april19. pdf

38. Nunavik Department of Public Health. Call for Vigilance: Syphilis. 2017 January 2017;5(1). https://nrbhss.ca/sites/default/ files/Info-Mado\%20de\%20la\%20DSP\%20Nunavik\%20-\%20vol.\%20 5\%2C\%20no\%201\%20-\%20Call\%20for\%20vigilance\%20Syphillis.pdf

39. Fraser Health. Interim guidance on perinatal syphilis screening - June 2019. BC: FH; 2019. https://www. fraserhealth.ca/employees/medical-health-officer-updates/ interim-guidance-on-perinatal-syphilis-screening-june-2019\#.XZTMwIVKipq

40. Manitoba Health, Seniors and Active Living. RE: Congenital Syphilis in Manitoba. Winnipeg (MB): $\mathrm{MH}$; February 25, 2019. https://www.gov.mb.ca/health/ publichealth/cdc/docs/hcp/2019/022519.pdf

41. Northwestern Health Unit. Medical Alert - Syphilis Update. Ontario; NWHU; March 12, 2019. https://www.nwhu. on.ca/Audiences/Documents/Medical\%20Alert\%20-\%20 Syphilis\%20Update\%2003.12.19.pdf

42. Reproductive Care Program of Nova Scotia. Re: Public Health Alert: Syphilis Outbreak. January 20, 2020. http://rcp.nshealth.ca/sites/default/files/clinical-practiceguidelines/ProviderLetter_Syphilis_2020_01_20.pdf

43. Chan EY, Smullin C, Clavijo S, Papp-Green M, Park E, Nelson M, Giarratano G, Wagman JA. A qualitative assessment of structural barriers to prenatal care and congenital syphilis prevention in Kern County, California. PLoS One 2021;16(4):e0249419. DOI PubMed
44. Fukuda A, Katz AR, Park IU, Komeya AY, Chang A, Ching N, Tomas JE, Wasserman GM. Congenital syphilis: a case report demonstrating missed opportunities for screening and inadequate treatment despite multiple healthcare encounters during pregnancy. Sex Transm Dis 2021;48(9):e124-5. DOI PubMed

45. Hayeems RZ, Campitelli M, Ma X, Huang T, Walker M, Guttmann A. Rates of prenatal screening across health care regions in Ontario, Canada: a retrospective cohort study. CMAJ Open 2015;3(2):E236-43. DOI PubMed

46. Mill J, Singh A, Taylor M. Women in the Shadows; Prenatal Care for Street-Involved Women. Can J Urban Res 2012;21(2):68-89. https://go.gale.com/ps/i.o?p=AONE\&u=g ooglescholar\&id=GALE|A337718414\&v=2.1\&it=r\&sid=AON E\&asid=17a9dc52

47. Singh A, Romanowski B. The return of syphilis in Canada: A failed plan to eliminate this infection. JAMMI 2019;4(4):215-7. https://jammi.utpjournals.press/doi/ full/10.3138/jammi.2019-08-22

48. National Collaborating Centre for Infectious Diseases. Plains Speak on STBBls, 2019 An emergent challenge for the prairies. Winnipeg (MB): NCCID; 2019. https://nccid.ca/ publications/plains-speak-on-stbbis-2019/

49. Cook JL, Green CR, de la Ronde S, Dell CA, Graves L, Morgan L, Ordean A, Ruiter J, Steeves M, Wong S. Screening and Management of Substance Use in Pregnancy: A Review. J Obstet Gynaecol Can 2017;39(10):897-905. DOI PubMed

50. Centre de collaboration nationale des maladies infectieuses. Atteindre les populations mal desservies: Tirer parti des tests de dépistage au point de service des infections transmissibles sexuellement et par le sang pour explorer de nouvelles options de programme au Canada. Winnipeg (MB) : CCNMI; 2018. https://ccnmi.ca/publications/ atteindre-les-populations-mal-desservies-tirer-parti-destests-de-depistage-au-point-de-service-des-infectionstransmissibles-sexuellement-et-par-le-sang-pour-explorerde-nouvelles-options-de-progra/

51. National Collaborating Centre for Infectious Diseases. Point of Care Testing for Sexually Transmitted and Blood-Borne Infections. Winnipeg (MB): NCCID; 2019. https://nccid.ca/ wp-content/uploads/sites/2/2019/11/Point-of-Care-Testingfor-Sexually-Transmitted-and-Blood\%E2\%80\%90BorneInfections-A-Canadian-Realist-Review.pdf

52. Santé Canada. Faits saillants sur les médicaments et les instruments médicaux 2020 : Instruments médicaux. Ottawa (ON) : SC; 2021. https://www.canada.ca/fr/sante-canada/ services/publications/medicaments-et-produits-sante/ faits-saillants-2020-medicaments-instruments-medicaux/ instruments-medicaux.html 
53. Agence de la santé publique du Canada. Blogue de données : Quelles sont les répercussions de la COVID-19 sur la prestation des services liés aux infections transmissibles sexuellement et par le sang (ITSS), y compris les services de réduction des méfaits, au Canada? Ottawa (ON) : ASPC; 2021. https://sante-infobase.canada.ca/labo-de-donnees/ covid-19-repercussions-services-itss.html

54. Pagaoa M, Grey J, Torrone E, Kreisel K, Stenger M, Weinstock H. Trends in Nationally Notifiable Sexually Transmitted Disease Case Reports During the US COVID-19 Pandemic, January to December 2020. Sex Transm Dis 2021;48(10):798-804. DOI PubMed

55. Simões D, Stengaard AR, Combs L, Raben D; EuroTEST COVID-19 impact assessment consortium of partners. Impact of the COVID-19 pandemic on testing services for HIV, viral hepatitis and sexually transmitted infections in the WHO European Region, March to August 2020. Euro Surveill 2020;25(47):2001943. DOI PubMed
57. Agence de la santé publique du Canada. Méfaits associés aux opioïdes et aux stimulants au Canada. Ottawa (ON) : ASPC; 2021. https://sante-infobase.canada.ca/mefaitsassocies-aux-substances/opioides-stimulants

58. Pan American Health Organization. Why Data Disaggregation is key during a pandemic. Washington (DC): PAHO; 2020. https://iris.paho.org/handle/10665.2/52002

59. Etowa J, Hyman I, Dabone C, Mbagwu I, Ghose B, Sano Y, Osman M, Mohamoud H. Strengthening the Collection and Use of Disaggregated Data to Understand and Monitor the Risk and Burden of COVID-19 Among Racialized Populations. Can Stud Popul 2021;48:1-16. DOI PubMed

60. Womersley K, Ripullone K, Peters SA, Woodward M. Covid-19: male disadvantage highlights the importance of sex disaggregated data. BMJ 2020;370:m2870. DOI PubMed

56. Sentís A, Prats-Uribe A, López-Corbeto $E$, Montoro-Fernandez M, Nomah DK, de Olalla PG, Mercuriali L, Borrell N, Guadalupe-Fernández V, Reyes-Urueña J, Casabona J, Catalan HI; Catalan HIV and STI Surveillance Group. The impact of the COVID-19 pandemic on Sexually Transmitted Infections surveillance data: incidence drop or artefact? BMC Public Health 2021;21(1):1637. DOI PubMed

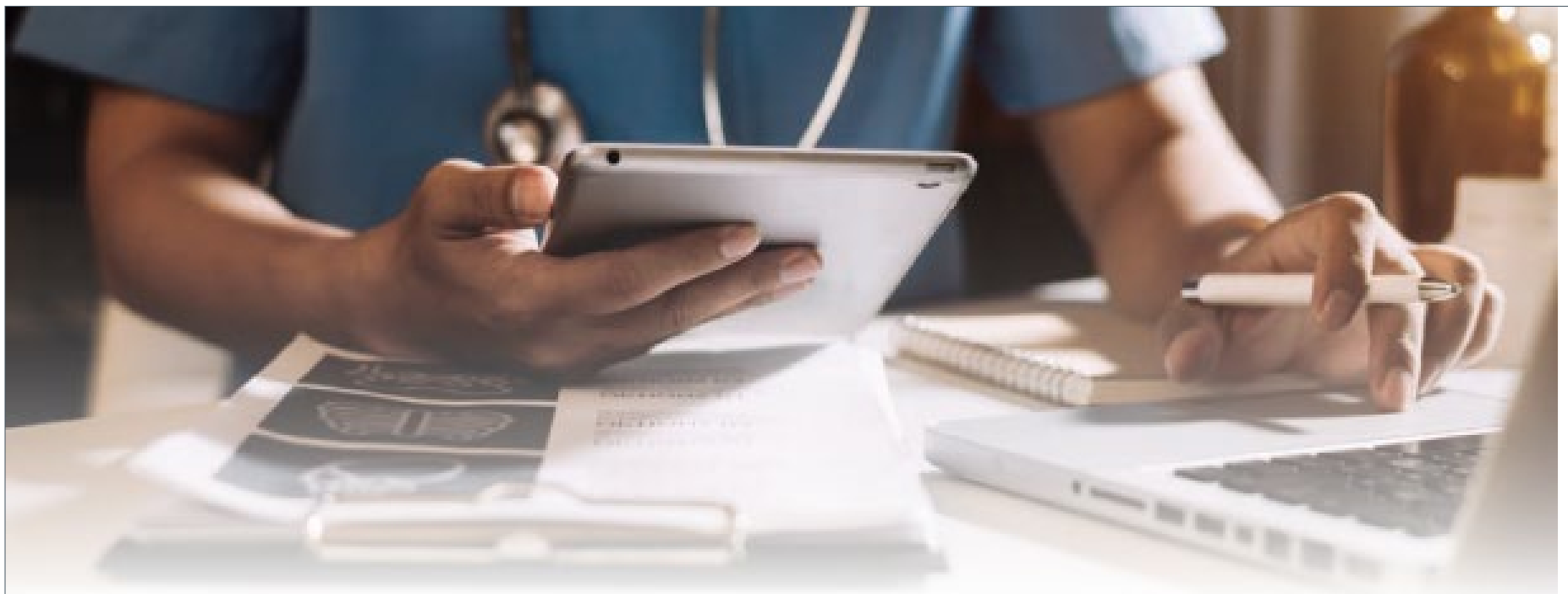

L'Agence de la santé publique du Canada remplace les Lignes directrices canadiennes sur les infections transmissibles sexuellement (LDCITS) par une série de guides conviviaux qui fournissent des informations essentielles et des recommandations de santé publique sur le dépistage, le traitement et le suivi des infections transmissibles sexuellement et par le sang (ITSS).

Vous pouvez accéder aux guides intitulés « Infections transmissibles sexuellement et par le sang : Guides à l'intention des professionnels de la santé " au

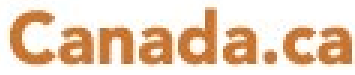

